\title{
Composition et métaphores identifiantes
}

\author{
Mireille Guittonneau et Sylvie Le Poulichet
}

Comme en témoigne l'œuvre freudienne, l'objectif de la psychanalyse fut longtemps de reconstituer une vérité historique, soit « une image fidèle des années oubliées par le patient, une image complète de toutes ses parties essentielles $»^{1}$. Ainsi, en prenant le pas sur la remémoration, dont il avait fallu constater qu'elle était parfois impossible, la reconstruction restait, en 1937, fondamentalement liée à la réalité événementielle. Mais, en mettant autant l'accent sur le «vrai », Freud ne cherchait-il pas, surtout, à établir la scientificité de la psychanalyse ? En effet, Freud semble se dégager de cet impératif de vérité, lorsqu'à la fin de son article, il écrit, au sujet de la force de conviction acquise par les constructions, en dehors de toute remémoration : "dans quelle condition cela a lieu et de quelle façon il est possible qu'un substitut aussi imparfait produise quand même un plein effet, c'est ce qui devra faire l'objet de recherches ultérieures. ${ }^{2} \gg$ Or, cette question n'est-elle pas essentielle ? En effet, elle nous livre le constat établi par Freud lui-même : si l'analyste est en quête de réalité historique, c'est pourtant autre chose qui se présente à lui. Et depuis, nombre d'analystes ont continué à s'interroger, cherchant à apporter des éléments de réponse à cette question. S. Viderman, en particulier, a ouvert la voie en soulignant que l'histoire à laquelle donne vie la construction de l'analyste ne peut se penser en dehors de l'espace et de la parole qui la fait advenir. « Ce qui de l'inconscient vient à notre connaissance n'est pas seulement médiatisé par le langage mais créé par le langage ${ }^{3}$.» La construction, dans ces conditions, lui apparaît comme «une invention ${ }^{4}$, témoignage de la faculté d'imagination créatrice de celui qui la formule ${ }^{5} »$, ayant, cependant, à coïncider avec la réalité vécue par le patient.

Ainsi, s'est peu à peu forgée l'hypothèse que le rôle de l'analyste était moins de retrouver une histoire perdue, enfouie dans les profondeurs de l'inconscient, que de faire advenir des images, des identifications aussi singulières qu'inédites. Dès lors, l'espace analytique est devenu un lieu de création. Mais pour interroger la créativité de l'analyste, ne fallait-il pas au préalable s'être dégagé, au moins en partie, d'une représentation de la cure selon laquelle

\footnotetext{
${ }^{1}$ Freud S., (1937), «Constructions dans l'analyse », dans Résultats, idées, problèmes, T. II, PUF, 1992, p. 270.

${ }^{2}$ Ibid. p. 273.

${ }^{3}$ Viderman, S., La construction de l'espace analytique, Paris, Gallimard, 1970, p. 63.

${ }^{4} \mathrm{Au}$ sens où l'on est l'inventeur d'un trésor.

${ }^{5}$ Ibid. p. 65.
} 
« deux pièces entièrement distinctes, (qui) se jouent sur deux scènes séparées et concernent deux personnages dont chacun est chargé d'un rôle différent. ${ }^{6} »$ ? Car l'évolution de la psychanalyse montre que c'est le travail clinique avec des patients aux fonctionnements limites, psychotiques, enfants ou adultes, qui a tout à la fois interrogé une telle représentation de la cure et sollicité la créativité de l'analyste. C'est, en effet, dans ces champs cliniques que les analystes ont dû inventer de nouveaux outils théoriques et techniques permettant à la parole de leurs analysants de prendre place et de donner lieu à une élaboration psychique. Démarche qui s'inscrit, alors, dans une double exigence : prendre en compte la singularité de ce patient là, nécessitant une approche différente, sans perdre de vue les spécificités de la démarche analytique comme, par exemple, la dissymétrie des positions entre analyste et analysant.

Comment s'exprime alors cette créativité ? Quels mouvements la composent ? Nous ferons l'hypothèse qu'avec certains patients, il est fondamental que l'analyste réalise un travail de composition par lequel peuvent notamment se dénouer des identifications mortifères, tandis que le temps et l'espace peuvent s'élaborer, témoignant du déploiement d'un espace-temps interne. Nous interrogerons également le processus en jeu à partir de la notion de métaphores identifiantes qui nous conduira sur la voie d'un miroir paradoxal.

Il s'agit donc, dans cet article, à la fois de préciser certains des mouvements psychiques qui se produisent dans la cure et qui engagent les deux psychés en présence et de souligner le rôle fondamental des figurations forgées par l'analyste, sans lesquelles, bien souvent, le processus analytique ne peut se déployer.

On sait que le latin figura est formé sur le radical de fingere «modeler dans l'argile » et qu'il possède de multiples sens : forme, aspect, représentation sculptée, mode d'expression, signe, symbole. Et «figurer» (dérivé de figura), c'est d'abord façonner, donner une forme, représenter sous une forme visible ${ }^{7}$. Avec la rhétorique, la figure est définie comme un écart par rapport à une «valeur normale », à ce qui est appréhendé comme un sens propre au signe. La métaphore y est alors considérée comme l'exemple privilégié de la figure. Les figures, formes particulières de la représentation, sont essentiellement envisagées comme des métaphores, c'est-à-dire ce qui fait image par substitution ${ }^{8}$. Aussi la présentation

\footnotetext{
${ }^{6}$ Freud S., (1937), «Constructions dans l'analyse », op. cit., p. 270.

${ }^{7}$ Rey, A. (1992). Dictionnaire historique de la langue française, Le Robert.

${ }^{8}$ Jakobson, R. (1963). Essais de linguistique générale. Ed. de Minuit.
} 
(Darstellung) de figures par l'analyste pourrait entre autres articuler de manière particulière le langage et l'image, produisant alors un travail de composition.

\section{1 - Compositions et devenirs}

Dans certaines cures ou psychothérapies, on découvre que des sujets ont été précocement figés dans des identifications inconscientes insoutenables, auxquelles ils cherchent, en vain, à échapper. Dans l'impossibilité de s'y reconnaître, ils sont pourtant constamment ramenés à cette image d'eux-mêmes qui ne saurait être à l'origine d'une construction subjectivante. Qu'il s'agisse, par exemple, du fantasme d'un «enfant donneur ${ }^{9}$, par lequel le sujet est amené à faire don à l'autre d'une partie de son corps, de sa psyché, ou du fantasme d'un enfant, meurtrier à venir de ses parents ${ }^{10}$, qui inscrit la relation naissante sous le sceau d'une lutte à mort, on réalise l'impasse dans laquelle ils se trouvent. Car, s'il leur faut se soumettre à ces identifications pour exister dans le regard de l'autre, c'est un reflet qui fait effraction, entraînant une dissolution, une désagrégation partielle de leur image spéculaire. L'effroi qui accompagne la rencontre d'une telle identification induit alors un état de sidération, privant le sujet du recours à une pensée mobile et féconde. Et c'est bien souvent par des fonctionnements paradoxaux qu'ils tentent d'échapper à ces identifications inconscientes : s'effacer pour exister, survivre en mimant la mort. Ces fonctionnements révèlent la position intenable dans laquelle ils sont enfermés, conduisant à une représentation du corps altérée, dépourvue de volume, intriquée à des formations fantasmatiques singulières, comme peuvent l'être les « chimères du corps ${ }^{11}$. De telles représentations entraînent ces sujets sur la voie d'un effacement des affects ${ }^{12}$ comme tentative de désidentification et de maintien d'une enveloppe psychique et corporelle menacée par chaque apparition, dans le miroir, du visage de l'enfant meurtrier ou de l'enfant mutilé. C'est alors une douleur hémorragique ou explosive qui s'exprime, témoin de l'éclatement du miroir, tandis qu'un recours à la sensorialité s'impose parfois pour en restaurer l'unité. Ecrasées par de telles identifications, les représentations du temps et de l'espace semblent également mises en suspens - en attente et en souffrance - tandis qu'à d'autres moments prédominent des mouvements de disparition, d'effacement du temps et de l'espace, entravant les possibilités d'une inscription psychique

\footnotetext{
${ }^{9}$ Le Poulichet S., Les chimères du corps. De la somatisation à la création, Paris, Flammarion, 2010.

${ }^{10}$ Guittonneau M., «Identification narcissique, figure du double et fantasme d'un enfant meurtrier », article à paraître, dans Cliniques méditerranéennes, L'enfant et ses fantômes, 2012.

${ }^{11}$ Le Poulichet S., Les chimères du corps. De la somatisation à la création, op. cit.

${ }^{12}$ Guittonneau M., Les fonctions du contre-transfert dans la figuration de la détresse, thèse soutenue le $1^{\mathrm{er}}$ juillet 2011, Paris 7, D. Diderot.
} 
des événements. Et les mots de ces patients semblent, tout d'abord, s'immobiliser, faute de pouvoir exprimer une détresse sans immédiatement être assailli par elle; les mots ne parvenant pas, alors, à creuser le moindre écart. Car, en collant à la peau, les mots ne peuvent que redoubler l'effroi originel associé à la détresse produite par ces positions identificatoires aliénantes.

La relation transférentielle, par conséquent, se trouve elle aussi marquée par des mouvements paradoxaux, lorsque, par exemple, un sujet semble prendre la fuite pour s'extraire d'une relation soudain inquiétante, alors qu'en fait, tout son être est en attente de cet autre, l'analyste, et de sa capacité à faire de sa détresse autre chose qu'une réalité crue. La position prise par l'analyste est alors déterminante pour l'engagement et la poursuite de la cure. En effet, si la créativité de l'analyste, comme capacité à présenter au sujet des figurations singulières, insolites et parfois même énigmatiques, prend une importance majeure avec certains patients, c'est qu'à travers elle, se révèle aussi le mouvement désirant qui anime l'analyste. Car c'est bien ce mouvement désirant qui nourrit sa capacité à laisser résonner en lui des matériaux psychiques étranges. Mouvement vers l'autre et reconnaissance de sa singularité, le désir de l'analyste donne lieu à des figurations, créatrices d'un espace psychique partagé, sorte de fil tendu entre son patient et lui, auquel le premier peut, pour un temps, se tenir. En effet, la présentation de ces figurations, traces de la résonance et du désir de l'analyste, apparaît comme la condition pour que certains analysants puissent faire face à la terreur provoquée par le surgissement de ces identifications mortifères. Ainsi, se dégage le travail de composition qui, parfois, se réalise dans la cure et qui renouvelle la compréhension $\mathrm{du}$ processus analytique et de ses enjeux. En effet, ce sont les transferts qui sont à l'œuvre dans cette «composition psychique mouvante où les mouvements psychiques de l'analyste et ceux de l'analysant cheminent ensemble. ${ }^{13}$ »

Dès lors, il s'agit, par ce travail de composition de rendre possible un double mouvement de déliaison et de liaison par lequel peuvent se défaire des identifications aliénantes et se recomposer de nouvelles figures. L'enjeu concerne alors des devenirs-sujet ${ }^{14}$, multiples et toujours en mouvements, témoignant qu'un sujet ne peut qu'être en devenir. En effet, il convient de se dégager de l'idée selon laquelle le processus analytique permettrait de faire apparaître un sujet. Car si l'on tient compte de la division interne mise en évidence par la

\footnotetext{
${ }^{13}$ Le Poulichet S., Les chimères du corps, de la somatisation à la création, op. cit. p. 96.

${ }^{14}$ ibid, chap. 3.
} 
psychanalyse, prétendre à la découverte d'un sujet ne peut qu'être un leurre. En étant luimême un processus, le travail de composition témoigne alors de ce mouvement incessant, à la fois en donnant forme aux différentes identifications, parfois composites, qui habitent l'analysant et en rendant possible un processus de recomposition, suffisamment labile pour n'être plus l'expression d'une désignation surmoïque figée en un «tu es ceci».

Comment se produisent dans la cure ces devenirs-sujets ? Loin de chercher à établir, à reconstruire une fois pour toutes, l'histoire de l'analysant, il s'agit pour l'analyste de lui présenter des «images-événements » ${ }^{15}$, c'est-à-dire des figurations qui font surgir des liens inédits, n'ayant jusqu'alors pas été pensés. Et c'est en laissant résonner les paroles de l'analysant avec les images qui naissent en lui que la parole de l'analyste parvient à déployer des compositions venant «penser-figurer le corps et toucher le corps par la voie du signifiant ${ }^{16} »$. Alors ces «images-événements ${ }^{17}$ qui engagent «le corps, ses ouvertures et fermetures, ses effractions, ses formations et déformations ${ }^{18} »$, permettent à l'analysant de s'identifier et de nouer une nouvelle relation à son corps. Se produit ainsi « une prise de $\operatorname{corps}^{19} »$, c'est-à-dire un nouage de sensations, de pulsions, d'images et d'éléments du langage permettant au Je de se projeter au niveau corporel comme un volume, tandis que se créée dans le champ du langage la possibilité de se situer comme Je. C'est là ce qui permet de dissoudre les compositions fantasmatiques que sont les chimères et d'instituer un nouveau « jugement d'existence ${ }^{20} »$.

Le travail de composition réalisé par l'analyste et par l'analysant peut également être pensé sur le modèle du rêve qui, en traitant les mots comme les choses, est le lieu privilégié de création de telles «images-événements». Les images du rêve évoquées dans la cure par le patient lui-même doivent alors être entendues dans leur fonction souvent essentielle de création de liens, capables de faire apparaitre les identifications inconscientes qui les saisissent. Ainsi, par la révélation de liens inouïs, apparaît une dimension essentielle du processus conduisant aux devenirs-sujet : il se réalise par des devenirs-autre, touchant autant l'analyste que l'analysant. Impossible, par conséquent, d'ignorer les effets de surprise qui se produisent à certains moments dans une cure. Au contraire, ils s'avèrent essentiels, témoins de

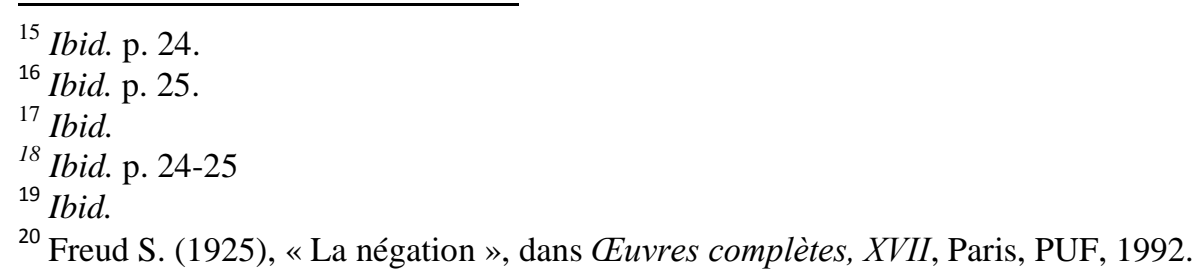


ces devenirs-autre. En effet, l'expérience subjectivante des devenirs-autre a à voir avec «la capacité de l'analyste de donner ce qu'il n'a pas ${ }^{21}{ }$. Et 1 'on peut dire que «l'analyste intervient souvent dans un sens dont il ne se savait pas capable : il s'agit d'une potentialité qu'il ne sait pas avoir mais qu'il va découvrir dans sa parole ${ }^{22} \gg$. Pour autant, ce processus n'a rien d'évident et ne manque pas de susciter diverses résistances de contre-transfert, en particulier des résistances à se laisser déformer et interpréter par le patient. En effet, si la capacité à se laisser surprendre ouvre sur la créativité et l'ouverture à l'inattendu, la non préparation donne également au fait d'être surpris une potentialité traumatique. Et pourtant, l'inattendu doit parfois surgir en l'analyste pour survenir ensuite chez l'analysant. Ainsi se créé un «dispositif spéculaire ${ }^{23} »$, soit une «surface de réflexion (au double sens du terme) habitée par les dimensions de la voix et du regard, qui renvoie à l'analysant la pluralité de ses messages, en leur permettant de résonner et d'apparaître en de nouvelles compositions ${ }^{24}{ }^{»}$, ce qui fait de l'analyste, entre autres choses, un miroir sonore. Alors quelque chose se produit, un récit peut advenir, témoin de cette création commune que sont, en définitive, ces figurations.

\section{2 - L'effroi et les métaphores identifiantes}

Le processus en jeu dans ce travail de composition peut également être éclairé par la notion de métaphores identifiantes; les métaphores étant l'une des formes qu'il emprunte. En effet, on s'aperçoit que la présentation par l'analyste d'une métaphore, grâce à sa capacité à introduire une distance, rend possible, pour certains sujets, l'élaboration d'un vécu d'effroi. Mais c'est aussi cette qualité qui montre l'existence d'un paradoxe au cœur même de ce travail de figuration : c'est en faisant écart, en déformant d'une certaine manière un vécu de détresse infinie, qu'une métaphore le révèle et crée de nouvelles possibilités identificatoires.

N'est-ce pas ce que Jorge Semprun interrogeait lorsqu'il écrivait, au sujet du récit d'expériences telles que celles des camps de concentration: «Un doute me vient sur la possibilité de raconter. Non pas que l'expérience vécue soit indicible. Elle a été invivable, ce qui est tout autre chose. $»^{25}$ ? Et selon lui, seul l'art pouvait permettre à l'auditeur d'approcher la réalité de cette expérience effroyable. «Raconter bien, ça veut dire : de façon à être entendus. On n'y parviendra pas sans un peu d'artifice. Suffisamment d'artifice pour que ça

\footnotetext{
${ }^{21}$ Merleau-Ponty M. «L'œil et l'esprit », Les temps modernes, n 184-185, 1961.

${ }^{22}$ Le Poulichet S., Les chimères du corps, de la somatisation à la création, op. cit. p. 73.

${ }^{23}$ Le Poulichet S., Psychanalyse de l'informe, Paris, Flammarion, 2003, p. 43.

${ }^{24}$ Le Poulichet S., Les chimères du corps, de la somatisation à la création, op. cit.. p. 62.

${ }^{25}$ Semprun J., L'écriture ou la vie, Paris, Gallimard, 1994, p. 25.
} 
devienne de l'art. (...) Comment raconter une vérité peu crédible, comment susciter l'imagination de l'inimaginable, si ce n'est en élaborant, en travaillant la réalité, en la mettant en perspective ? Avec un peu d'artifice, donc ! ${ }^{26} \mathrm{Si}$, selon Semprun, c'est le sujet qui doit, en premier lieu, se livrer à telle opération, la question se pose cependant d'une capacité pour tous à se livrer à une telle création. Certains sujets n'ont-ils pas besoin de s'appuyer sur les mots d'un autre qui occupe, pour un temps, la fonction de «porte-parole ${ }^{27}$ ayant à apporter au récit cet artifice, seule façon d'en faire une œuvre de passage ? N'est-ce pas cela que les métaphores proposées par l'analyste parviennent à réaliser ?

En effet, en empruntant la voie des métaphores, le vécu d'effroi de ces patients, resté jusqu'alors en dehors des mots, peut prendre forme. Plus exactement, il peut prendre une forme autre qui permet donc de l'envisager, d'en contempler le reflet sans être saisi par la terreur. Figuration et défiguration semblent donc travailler de concert, témoignant que les métaphores ne sauraient être assimilées à la photographie d'une histoire passée. Car l'écart qui les constitue ne peut situer les métaphores dans le registre de l'identique. Au contraire, cet écart, le jeu qui l'accompagne, se trouvent redoublés par le fait que l'analyste participe à la formation de ces figurations métaphoriques. En effet, ses propres matériaux psychiques conscients, préconscients et inconscients se mêlent à l'expérience livrée par l'analysant, pour créer ces figurations composites, leur donnant leur caractère parfois surprenant et énigmatique. C'est bien là, la part de l'autre qui s'impose, constitutif d'un miroir paradoxal faisant œuvre d'un écart entre le sujet et sa détresse. Ainsi, à travers la visée paradoxale de ces métaphores, se joue un accès à l'altérité, en soi et en l'autre. Par conséquent, en faisant apparaître une image de soi étrange, qui résonne avec une expérience vécue, sans être pour autant dans le registre du «vrai », qui évoque et esquisse un reflet sans prétendre à l'exactitude, les métaphores présentées par l'analyste permettent à ces patients d'initier un traitement psychique de leurs expériences infantiles douloureuses. Alors, peuvent se défaire certaines identifications insoutenables, tandis qu'apparaît dans le miroir un reflet dans lequel il est désormais possible de se reconnaître sans en être aussitôt effacé.

\section{3 - Paradoxes et métaphores}

Si la fonction paradoxale des métaphores nous paraît essentielle, il faut cependant souligner qu'elle a été fort peu mise en avant. En effet, ce sont bien davantage leurs mécanismes qui ont

\footnotetext{
${ }^{26}$ Ibid. p. 166.

${ }^{27}$ Aulagnier P. (1975), La violence de l'interprétation, Paris, PUF, 1995.
} 
retenu l'attention, aux premiers rangs desquels on trouve le déplacement et la condensation. Ainsi, lorsque l'on interroge la signification étymologique ${ }^{28} \mathrm{du}$ terme «métaphore », on découvre que ce mot, issu du latin metaphora, lui-même emprunté au grec metaphora «transport » renvoie à l'idée de transfert et de déplacement. Dans le champ littéraire, il s'agit donc d'un «procédé par lequel on substitue un terme à un autre pour produire une image ${ }^{29}$; le passage d'un registre à un autre venant alors enrichir la signification initiale. Ainsi, la fonction paradoxale des métaphores se trouve-t-elle maintenue dans l'ombre, mais reconnaissable pour qui veut bien y porter le regard. De même, n'est-ce pas cette dimension paradoxale qui apparaît à demi mots dans la réflexion de Lacan, lorsqu'il interroge le fait que "le langage ait son maximum d'efficacité quand il arrive à dire quelque chose en disant autre chose $»^{30}$ ? Pourtant, il centre son élaboration sur l'identification qui, selon lui, structure les métaphores, faisant apparaître trois caractéristiques déterminantes. D'une part, explique-til, une métaphore repose sur «un transfert de signifiés », ${ }^{31}$ c'est-à-dire que ce sont ses significations latentes, symboliques, qui sont essentielles et qui lui donnent ses potentialités dynamiques. D'autre part, souligne-t-il, c'est la similarité de position dans la phrase qui donne aux métaphores leur dimension identificatoire. Enfin, elles témoignent de l'existence possible d'une déconnexion entre le signifiant et sa signification ${ }^{32}$. Et ce, grâce à l'existence de la syntaxe qui permet de différencier, de ne pas réduire le sujet à ses qualités. "La métaphore suppose qu'une signification est la donnée qui domine et qu'elle infléchit, commande l'usage du signifiant, si bien que toute espèce de connexion préétablie, je dirais lexicale, se trouve dénouée. ${ }^{33}$. Ainsi, à partir de ces différents extraits et références, peut-on faire l'hypothèse que, dans le travail de composition, les processus primaires constitutifs des métaphores sont mis au service d'un but paradoxal. En effet, le déguisement qui recouvre certains vécus infantiles, certaines identifications inconscientes est, dans ce champ clinique, un déguisement nécessaire, destiné à révéler, à rendre visible et non à masquer. N'est-ce pas, alors, ce double registre d'une visée paradoxale, portée par les processus primaires, qui permet à ces analysants de progressivement s'inscrire dans un travail de liaison, délaissant un fonctionnement organisé par une forme de destructivité pour laisser place au conflit intrapsychique et au refoulement, moins invalidants? Le recours enfin possible aux

\footnotetext{
${ }^{28}$ Dictionnaire étymologique et historique de la langue française, Paris, Le livre de poche, 1996.

${ }^{29}$ Vocabulaire du commentaire de texte, Paris, Larousse, 1993.

${ }^{30}$ Lacan J., « Métaphore et métonymie II : articulation signifiante et transfert du signifié », dans Le séminaire, Livre III, Paris, Le Seuil, 1981, p. 255.

${ }^{31}$ Lacan J., « Métaphore et métonymie II : articulation signifiante et transfert du signifié », op. cit. p. 258.

${ }^{32} \mathrm{P}$. Fédida parlera de la dé-signification qui marque toute métaphore.

${ }^{33}$ Lacan J., « Métaphore et métonymie I : sa gerbe n'était point avare, ni haineuse », op. cit. p. 248.
} 
métaphores paradoxales apparaîtrait alors comme le premier pas vers un «traitement psychique $^{34} \gg$ de ce vécu désubjectivant, permettant à l'analysant de cesser d'être aspiré par une image effroyable à laquelle il était jusqu'alors condamné à s'identifier de façon absolue.

\section{4 - Donner corps aux mots}

Toutefois, le travail de liaison permis par les métaphores n'engage pas seulement la sphère des représentations mais concerne tout autant les affects. En effet, un lien étroit unit les métaphores aux affects, qu'elles ont la capacité de faire naître. Voici ce que disent, à ce sujet, les commentaires littéraires : «La métaphore révèle la face cachée du monde en soulignant des analogies profondes entre les êtres et les choses. Très expressive, elle permet de donner corps à des idées et de traduire visuellement des sensations ou des sentiments. Procédé inépuisable d'enrichissement de la langue, elle s'accompagne d'effets poétiques et dramatiques qui éveillent chez le lecteur des émotions profondes. $»^{35}$. Or, bien souvent, ces analysants ressentent tout mouvement affectif comme un danger menaçant leur intégrité psychique et corporelle. En faisant surgir des affects qui ne fassent pas voler en éclats leurs frêles limites, les métaphores sont l'occasion d'une expérience nouvelle par laquelle peuvent se tisser des liens inédits entre affects et représentations. Surprises, sources de curiosité, de plaisir ou de chagrin, on voit s'initier à chaque fois, un mouvement de réappropriation des affects, ce qui, dans l'histoire de ces patients constitue une reconquête décisive. Ce mouvement donne alors lieu à un possible jeu avec les mots, renouant avec un plaisir de penser qui s'inscrit dans un plaisir partagé, rendant possible un nouvel investissement libidinal de la relation à l'autre et de la relation à soi. Car les affects, portés par le désir de l'analyste et sa capacité à laisser résonner en lui ce que livre à son écoute l'analysant, habitent chaque métaphore. Ainsi peut progressivement s'inscrire l'existence d'une profondeur des mots, d'un espace psychique interne. En donnant corps aux mots, les métaphores révèlent aussi la primauté d'un corps, source des pulsions, qui permet à la psyché de se représenter ce qu'elle vit et ressent par les modèles qu'il lui fournit. Il semble alors que les métaphores, en inscrivant le corps au cœur des mots, en tissant des liens entre les affects, les images et les mots, donnent au miroir qu'elles dessinent, des qualités particulières en plus de celles, déjà évoquées, du miroir sonore.

\footnotetext{
${ }^{34}$ Freud S. (1890), « Traitement psychique », dans Résultats, idées, problèmes, T. I, Paris, PUF, 1991.

${ }^{35}$ Vocabulaire du commentaire de texte, op. cit.
} 
En effet, en donnant corps aux mots, les métaphores restituent également au regard sa dimension tactile, ce qui lui confère sa capacité à se faire enveloppe. Comment se constitue, à l'origine, cette qualité tactile du regard ? Cette question nous ramène à la fonction du regard de la mère. Winnicott a en effet souligné qu'avant la rencontre du petit enfant avec son image dans un miroir, source d'une intense jubilation, existait un préalable indispensable : le reflet renvoyé par le regard maternel. Il a ainsi montré la nécessité que ce regard réfléchisse à l'enfant une image de lui-même, et les conséquences sévères, sur le développement de l'enfant, d'un regard fermé, qui ne parle que de l'état psychique de la mère ${ }^{36}$.

Depuis, G. Haag ${ }^{37}$ a également souligné le rôle du regard maternel, associé à la tenue du dos, dans la constitution de mouvements d'interpénétration qui, ensuite, donnent à l'enfant un sentiment d'enveloppe. Mais là encore, pour que ces mouvements d'interpénétration du regard (qui redoublent l'interpénétration bouche/mamelon) puissent se mettre en place, encore faut-il qu'existe, en face, un regard accueillant et disponible. Le regard ne conserve-t-il pas alors ce premier lien à la tenue du dos, se dotant d'une dimension « tactile » qui participe à sa capacité à faire enveloppe? A l'inverse, lorsque ces mouvements d'interpénétration font défaut, le regard semble perdre sa fonction d'enveloppe et devient le lieu d'une effraction. Il est alors ressenti comme perçant, pénétrant ${ }^{38}$.

Soulignons, enfin, la capacité du regard à révéler les affects ; capacité qui fait de lui un lieu d'échange ou un lieu d'effroi. En effet, lorsque l'adulte est débordé par des affects qui ne cessent de le défigurer, son regard ne peut conserver - ou déployer - ses qualités intégratives. Le regard, assimilé aux affects qu'il exprime devient alors synonyme d'effraction et d'explosion. Ainsi découvre-t-on que pour certains analysants, il est fondamental de rencontrer la sensation tactile procurée par le regard d'autrui, avant de pouvoir se risquer dans un regard direct.

\footnotetext{
${ }^{36}$ Winnicott D.W., « Le rôle de miroir de la mère et de la famille dans le développement de l'enfant », dans Jeu et réalité, Paris, Gallimard, 2000.

${ }^{37}$ Haag G., « Réflexions sur quelques jonctions psycho-toniques et psycho-motrices dans la première année de la vie. », Neuropsychiatrie de l'enfance, 1988, 36 (1), 1-8.

${ }^{38}$ En outre, le regard de l'enfant lui-même ne peut passer, alors, du stade où il s'agrippe à celui où il renvoie à l'autre, témoignage d'un échange à l'œuvre. Ainsi, E. Bick, dans «Considérations ultérieures sur la fonction de la peau », Les écrits de M. Harris et d'E. Bick, Larmor plage, les éd. du Hublot, 1998, p. 151, a-t-elle évoqué la tendance de certains enfants à "s'agripper avec les yeux et les oreilles comme de peau à peau ", s'inscrivant ainsi dans un fonctionnement où seule la bidimensionalité est reconnue ; agrippement qui vise un objet externe (lumière, voix) pour se sentir tenu et rassemblé. Parfois, le regard se fait, aux yeux de l'observateur, regard aveugle.
} 
Dès lors, ne peut-on penser que les métaphores, et plus largement les figurations proposées par l'analyste, constituent un regard unifiant qui allie une image mise en mots, la sensation d'être tenu et la possibilité d'accueillir en soi différents affects? En donnant corps et affects aux mots, les métaphores seraient donc, dans le champ du langage, l'équivalent du regard dans ses multiples dimensions (réflexive, émotionnelle et tactile). Ainsi, en intégrant la sensation d'être tenu, associée à l'enveloppement du regard, ces figurations venues de l'analyste concourraient-elles à faire limite, acquérant ainsi leur fonction identifiante. On a alors l'impression que ces figurations, comme les interpénétrations qui nourrissent les échanges, se constituent tout à la fois comme noyau, à partir duquel peuvent se mettre en place les processus primaires, donnant lieu à de nouvelles figurations élaborées désormais par le sujet lui-même, et comme enveloppe écartant le péril d'une déchirure toujours à venir. Mais c'est aussi parce qu'elles sont langage, langage venu d'un autre, qu'elles offrent au sujet la possibilité de faire une expérience équivalente à celle de l'enfant devant le miroir. Ainsi peuvent se tisser, se renouer ce qui ressort de l'imaginaire et du symbolique.

\section{Conclusion}

L'exigence de créativité qui s'impose avec certains patients surgit parfois sous la forme d'un travail de composition. Loin d'une reconstitution historique, ce travail de composition consiste à présenter des images-événements par lesquelles de nouvelles identifications, de nouveaux liens au corps peuvent se tisser, tandis que se dénouent des identifications inconscientes aliénantes. Ainsi s'engage un processus mettant en jeu des devenirs-sujet, toujours en recréation. L'une des formes prise par ce travail de composition, les métaphores, fait apparaître l'existence d'un paradoxe au cœur de ce processus : c'est en déformant, en voilant qu'elles révèlent certaines identifications mortifères ou certaines expériences d'effroi, restées jusqu'alors dans l'ombre et le silence. Fruits de la capacité de l'analyste à laisser résonner en lui les paroles livrées à son écoute par l'analysant, ces métaphores apparaissent bel et bien comme une œuvre commune, parfois étrange. Elles suscitent ainsi un mouvement de pensée, de réappropriation des affects non élaborés, tout en donnant corps au mots. Par conséquent, se dégage la dimension identifiante des métaphores qui, en liant des images mises en mots, la sensation d'être tenu et la possibilité d'accueillir en soi des affects, constitue, dans le champ du langage, un regard enveloppant au lieu d'un regard qui efface.

\section{Bibliographie}


Aulagnier P. (1975), La violence de l'interprétation, Paris, PUF, 1995.

Bick E., «Considérations ultérieures sur la fonction de la peau », dans Les écrits de M. Harris et d'E. Bick, Larmor plage, les éd. du Hublot, 1998.

Dictionnaire étymologique et historique de la langue française, Paris, Le livre de poche, 1996.

Freud S. (1890), «Traitement psychique », dans Résultats, idées, problèmes, T. I, Paris, PUF, 1991.

Freud S. (1925), «La négation », dans Euvres complètes, XVII, Paris, PUF, 1992.

Freud S. (1937), «Constructions dans l'analyse », dans Résultats, idées, problèmes, T. II, PUF, 1992.

Guittonneau M., Les fonctions du contre-transfert dans la figuration de la détresse, thèse soutenue le $1^{\text {er }}$ juillet 2011, Paris 7, D. Diderot.

Guittonneau M., «Identification narcissique, figure du double et fantasme d'un enfant meurtrier », article à paraître, dans Cliniques méditerranéennes, L'enfant et ses fantômes, 2012.

Haag G., «Réflexions sur quelques jonctions psycho-toniques et psycho-motrices dans la première année de la vie. », Neuropsychiatrie de l'enfance, 1988, 36 (1), 1-8.

Lacan J., «Métaphore et métonymie I : sa gerbe n'était point avare, ni haineuse », dans $L e$ séminaire, Livre III, Paris, Le Seuil, 1981.

Lacan J., « Métaphore et métonymie II : articulation signifiante et transfert du signifié », dans Le séminaire, Livre III, Paris, Le Seuil, 1981.

Le Poulichet S., Psychanalyse de l'informe, Paris, Flammarion, 2003.

Le Poulichet S., Les chimères du corps. De la somatisation à la création, Paris, Flammarion, 2010 .

Merleau-Ponty M. «L'œil et l'esprit », Les temps modernes, $\mathrm{n}^{\circ}$ 184-185, 1961.

Semprun J., L'écriture ou la vie, Paris, Gallimard, 1994.

Viderman, S., La construction de l'espace analytique, Paris, Gallimard, 1970.

Vocabulaire du commentaire de texte, Paris, Larousse, 1993.

Winnicott D.W., «Le rôle de miroir de la mère et de la famille dans le développement de l'enfant », dans Jeu et réalité, Paris, Gallimard, 2000.

\section{Composition et métaphores identifiantes}

\section{Résumé}


La créativité de l'analyste s'exprime avec certains patients par un travail de composition consistant à présenter des images par lesquelles s'engage un processus identificatoire singulier. L'une des formes prise par ce travail de composition - les métaphores - fait apparaître l'existence d'un paradoxe au cour du processus : c'est en déformant, en voilant qu'elles révèlent certaines identifications mortifères ou certaines expériences d'effroi. En constituant, dans le champ du langage, l'équivalent d'un regard enveloppant, au lieu d'un regard qui efface, elles témoignent de leur fonction identifiante.

Mots clefs: travail de composition, métaphores identifiantes, paradoxe, processus idenficatoire.

\section{Composition and identifying metaphors}

\section{Summary}

Psychoanalyst creativity appears with some patients by composition work. This work consists in suggesting images which make it able to create singulars identifications. One form of this composition work - the metaphors - exposes the existence of a paradox in this process : the metaphors reveal some alienating identifications, some terrors's experiences by distorting them. As they are, in language fields, the equivalent of a gazing eyes, instead of an erasing gaze, they testify their identifying function.

Keys words : composition work, identifying metaphors, paradox, identifying process. 